\title{
Um processo de Gamificação para o ensino superior: Experiências em um módulo de Bioquímica
}

\author{
Armando Maciel Toda ${ }^{1 *}$, Yuri Rafael Oliveira Silva², Wilmax Cruz ${ }^{1}$, Luciana \\ Xavier $^{2}$, Seiji Isotani ${ }^{*}$ \\ ${ }^{1}$ Instituto de Ciências Matemáticas e de Computação - Universidade de São Paulo \\ (USP) - Campus de São Carlos \\ Caixa Postal 668 - 13560-970 - São Carlos - SP - Brasil \\ ${ }^{2}$ Instituto de Ciências Biológicas - Universidade Federal do Pará (UFPA) - Campus \\ Básico - Rua Augusto Côrrea, 01. Guamá. \\ CEP 66075-110 - Belém - PA - Brasil \\ \{armando.toda, ynxs22, wilmaxmcruz, luxavier\}@gmail.com, \\ sisotanidicmc.usp.br
}

\begin{abstract}
Gamification is gaining prominence in the past few years due to various researches in many areas achieving positive results. In education, this concept has been widespread, yet it still lacks a systemic process to its implementation. This paper presents a conceptual model of a gamification process to be applied within higher education lessons. The main results of this work is a gamified process validated during the gamification of a biochemistry lesson in a Biotechnology course. To validate this process, we used the Intrinsic Motivation Inventory (IMI) to measure students' motivation after the lessons. Results show positive impact of gamification with stronger results in female students.
\end{abstract}

Resumo. A gamificação vem ganhando destaque nos últimos anos devido as inúmeras pesquisas realizadas em diversas áreas do conhecimento e os resultados positivos identificados pela comunidade. No ensino, este conceito vem sendo bastante difundido, no entanto, ainda há uma certa carência quanto a um processo sistemático para sua implantação. Este artigo apresenta um modelo conceitual de um processo de gamificação, para ser utilizado no ensino superior, tomando como base planos de aula e ementas. $O$ resultado principal foi um processo gamificado, aplicado em uma turma de Bioquímica do ensino superior, no curso de Biotecnologia. Para verificar a eficácia do modelo foi utilizado o Intrinsic Motivation Inventory (IMI) que mede a motivação dos discentes após o uso da gamificação. Os resultados mostram que o método foi considerado divertido e interessante pelos alunos, principalmente quanto aos participantes do gênero feminino, além de ter uma boa aceitação por parte do docente responsável.

\section{Introdução}

Os avanços tecnológicos permitiram ampliar a utilização das Tecnologias da Informação (TI) em diversas áreas. Dentre estas, a área de Educação e Ensino vêm se beneficiando por meio de sistemas e processos provindos da computação que possuem a capacidade de

* Os autores agradecem ao CNPq e a FAPESP (Processo 2016/02765-2) pelo apoio e financiamento deste projeto. 
auxiliar e aprimorar as metodologias pedagógicas existentes [De Paula et al. 2014; Isotani et al. 2009].Dentre estas tecnologias, pode-se citar o uso de jogos digitais e seus processos derivados, como a gamificação. Este conceito vem adquirindo destaque, por conta de dos vários resultados positivos alcançados [Borges et al. 2013]. No entanto ainda há uma forte carência quanto a eficiência individual dos elementos de gamificação utilizados e processos sistemáticos para implantação adequada na gamificação em ambientes de aprendizagem [Dicheva et al. 2015; Thiebes et al. 2014; Andrade et al., 2016].

Um estudo realizado recentemente [Mora et al. 2015] demonstrou a extensão de frameworks para implantar o processo de gamificação em diversas aplicações. No entanto, segundo os autores, ainda há a necessidade de desenvolver um modelo genérico, que agregue qualidade no processo. Além disso, também comentam sobre a discrepância na avaliação e validação dos frameworks apresentados.

Baseando-se no exposto, este trabalho propõe um método para mapear elementos de jogos, em planos de aula e ementas, e aplicá-los no ensino superior, sendo validado através do Intrinsic Motivation Inventory (IMI). Para apresentar o estudo, o trabalho está dividido em: sessão 2 apresenta os conceitos e trabalhos relacionados a gamificação no ensino; sessão 3 apresenta o método desenvolvido, assim como o protocolo do experimento; sessão 4 apresenta os resultados encontrados, realizando uma breve discussão; sessão 5 apresenta as conclusões e trabalhos futuros.

\section{Gamificação}

O processo de gamificação pode ser conceituado como a utilização de elementos de jogos (como mecânicas, dinâmicas e estética) fora de seu escopo original, com o intuito de motivar, engajar e melhorar a experiência de usuário em diferentes situações com atividades de treinamento e ensino [Deterding et al. 2011; Huotari and Hamari 2012; Kapp 2012; Zichermann and Cunningham 2011].

A partir do uso de motivadores extrínsecos, o processo afeta a motivação intrínseca do usuário, suportada pela Teoria da Autodeterminação (SDT). Esta teoria, segundo Deci e Ryan (1985), defende que a motivação é sustentada por 3 pilares, sendo: Autonomia, Competência e Relacionamento. A autonomia se baseia no indivíduo ter controle sobre suas ações, já a competência consiste no indivíduo adquirir conhecimento e habilidade sobre algo. Por fim, o relacionamento consiste na necessidade do indivíduo de se conectar com outras pessoas [Deci and Ryan 1985].

Estes 3 pilares podem ser encontrados em jogos digitais, por exemplo: a Autonomia pode ser representada pelo controle do jogador sobre as ações de seu avatar virtual; a Competência pode ser encontrada no progresso do usuário dentro do universo do jogo; o Relacionamento pode ser representado nas relações virtuais que o jogador se engaja, para continuar o jogo [Aparicio et al. 2012].

\section{Trabalhos Relacionados}

Dentre os trabalhos relacionados, pode-se citar o estudo realizado por [Hanus and Fox 2014]. Neste trabalho, os autores analisaram a aplicação da gamificação em um contexto de ensino, analisando performance acadêmica, motivação, comparação social, satisfação e esforço. Os autores explanam que a combinação de elementos gamificados utilizados contribuiu para um desempenho acadêmico abaixo do esperado, e não influenciou de forma significativa na motivação, engajamento, satisfação e comparação social dos 
discentes. No estudo realizado, eles associam a o rendimento à motivação intrínseca, que diminuiu durante a aplicação dos elementos de gamificação.

Outro trabalho interessante é o de [Mesquita et al. 2013, 2014], onde os autores desenvolveram uma metodologia gamificada para aulas de Linguagens e Técnicas de Programação, em um curso de Ciência da Computação. Neste, os autores obtiveram uma aceitação razoável dos elementos, mas não utilizaram métodos para medir a motivação ou performance dos indivíduos.

Além destes, pode-se citar também o trabalho de [Pedro et al. 2015], onde foi desenvolvido um ambiente de aprendizagem virtual e gamificado. Neste estudo, os autores explanam que os elementos de gamificação influenciaram de maneira positiva a performance dos participantes do gênero masculino, porém não foram encontrados resultados significativos em relação aos do gênero feminino.

\section{Método}

O método desenvolvido descreve 4 fases, sendo: Definição de conteúdo, Definição de Elementos de Jogos, Implantação e Validação. Na primeira fase, ocorre a análise dos planos de aula e ementas, elaborados pelo professor para aplicação da aula. Estes documentos foram escolhidos por fazerem parte do cotidiano do docente, e por conterem os conteúdos de forma organizada e sistemática [Takahashi and Fernandes 2004], auxiliando na geração de um mapa conceitual para a disciplina (Figura 1).

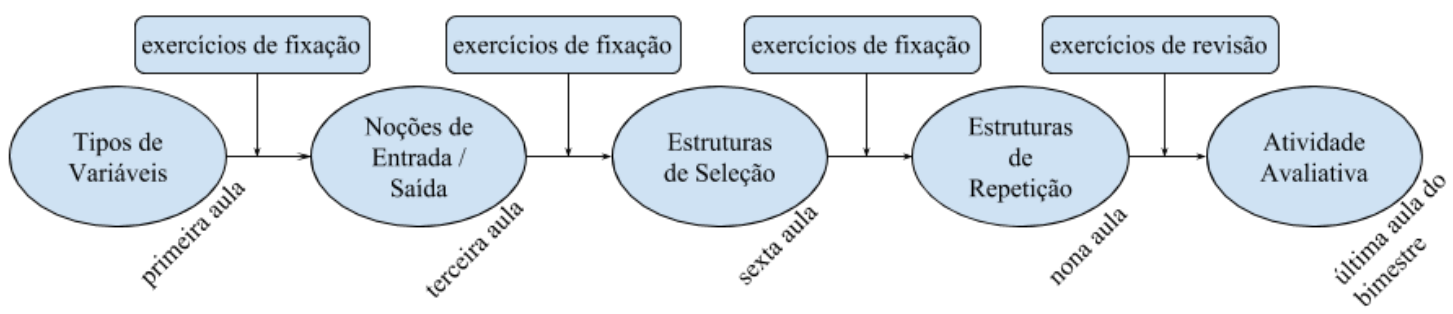

Figura 1: Exemplo de Mapa mental de uma disciplina de Noções de Programação Fonte: Autores (2016)

A Figura 1 apresenta um exemplo de mapa conceitual, gerado a partir de uma ementa, de um curso de Noções de Programação. Para geração do mapa, é importante considerar o conteúdo que será ministrado e as atividades vinculadas a este (definir ações). O mapa também é importante para auxiliar na definição dos elementos de jogos, que será realizada na Fase 2. Esta fase também é importante para definir o período em que cada conceito será ministrado pelo docente, auxiliando na organização da disciplina.

Em seguida, a Fase 2 define os elementos (mecânicas e dinâmicas) que serão utilizados. Para auxiliar o docente responsável na seleção, foi elaborado um conjunto de conceitos divididos em: Elementos de Feedback, consistem em motivadores extrínsecos aplicados a ação, e Elementos de Propriedade, que consistem nas características e objetivos da ação desenvolvida.

Estes elementos foram definidos a partir de pesquisas realizadas em revisões sistemáticas [Borges et al. 2013; Dicheva et al. 2015; Hamari et al. 2014] e do conjunto de elementos para jogos comportamentais desenvolvido por Dignan (2011). A listagem com a descrição dos elementos pode ser observada na Tabela 1: 
V Congresso Brasileiro de Informática na Educação (CBIE 2016)

Anais do XXII Workshop de Informática na Escola (WIE 2016)

Tabela 1: Divisão dos elementos

\begin{tabular}{|l|l|l|l|l|l|}
\hline \multicolumn{2}{|c|}{ Elementos de Feedback } & \multicolumn{3}{c|}{ Elementos de Propriedade } \\
\hline Reputação & Pontos & Conquistas & Cooperação & Renovação & Narrativa \\
\hline Troféus & Níveis & Progresso & Competição & Pressão & Economia \\
\hline \multirow{2}{*}{} & Decisões & $\begin{array}{l}\text { Atividades } \\
\text { Cognitivas }\end{array}$ & Oportunidades \\
\cline { 3 - 6 } & & Aleatoriedade & Segredos & Novidade \\
\cline { 3 - 6 } & &
\end{tabular}

A partir da combinação dos elementos presentes na Tabela 1, o docente responsável pode gerar diversas dinâmicas para serem aplicadas durante, ou todo, o período da disciplina. Para auxiliar na organização deste processo, foi gerada a Tabela 2.

Tabela 2: Tabela de Ações

\begin{tabular}{|l|l|}
\hline Nome da ação: & Data: \\
\hline Descrição: & \\
\hline Propriedades: & \\
\hline Feedback: & \\
\hline Comentários / Acompanhamento: & \\
\hline
\end{tabular}

Após a definição dos conceitos que serão abordados, ações e elementos de jogos, inicia-se a fase de Implementação. Esta consiste da aplicação das ações definidas na Fase 2. Para isso, é necessário um momento com os participantes da disciplina para explanação das regras operacionais que terão vigência durante todo o curso, as regras de atividades específicas podem ser explanadas neste momento, porém recomenda-se que sejam focadas apenas quando forem realizar a ação (de modo a não confundir o discente, num momento inicial).

Durante a fase de implantação, é recomendado ao docente que realize encontros para medição da satisfação da turma como um todo, a fim de evitar que discentes sejam penalizados por conta do processo. Por fim, é realizada a Fase 4, validação, por meio de questionários. A Tabela 3 apresenta a descrição das principais funções de cada fase.

Para validar o processo, é utilizado o Intrinsic Motivation Inventory (IMI), um instrumento de medição multidimensional que visa avaliar a experiência subjetiva do usuário, quanto a atividade realizada. Após coleta a análise das respostas, é possível planejar a próxima iteração, utilizando também os relatos dos alunos.

Tabela 3: Resumo de execução do método

\begin{tabular}{|l|l|}
\hline Fase & Descrição \\
\hline Definição dos Conteúdos & Analisar planos de aula e ementas \\
& Gerar mapa conceitual \\
& Definir tempo da disciplina \\
& Definir Ações \\
\hline
\end{tabular}


V Congresso Brasileiro de Informática na Educação (CBIE 2016)

Anais do XXII Workshop de Informática na Escola (WIE 2016)

\begin{tabular}{|l|l|}
\hline Definição dos Elementos de Jogos & $\begin{array}{l}\text { Definir Elementos de feedback das ações } \\
\text { Definir elementos de propriedade das } \\
\text { ações } \\
\text { Definir dinâmicas } \\
\text { Preencher Tabela de Ações }\end{array}$ \\
\hline Implantação & $\begin{array}{l}\text { Explanar as ações que serão } \\
\text { desenvolvidas } \\
\text { Explanar as regras envolvidas } \\
\text { Aplicar o método }\end{array}$ \\
\hline Validação & $\begin{array}{l}\text { Aplicar o IMI para avaliar a motivação do } \\
\text { discente } \\
\text { Coletar relatos } \\
\text { Analisar respostas para próxima iteração }\end{array}$ \\
\hline
\end{tabular}

\section{Resultados e Discussões}

Esta seção apresenta os resultados a partir da aplicação do método em uma disciplina modular de Bioquímica, no curso de Biotecnologia. Primeiramente foi realizada a

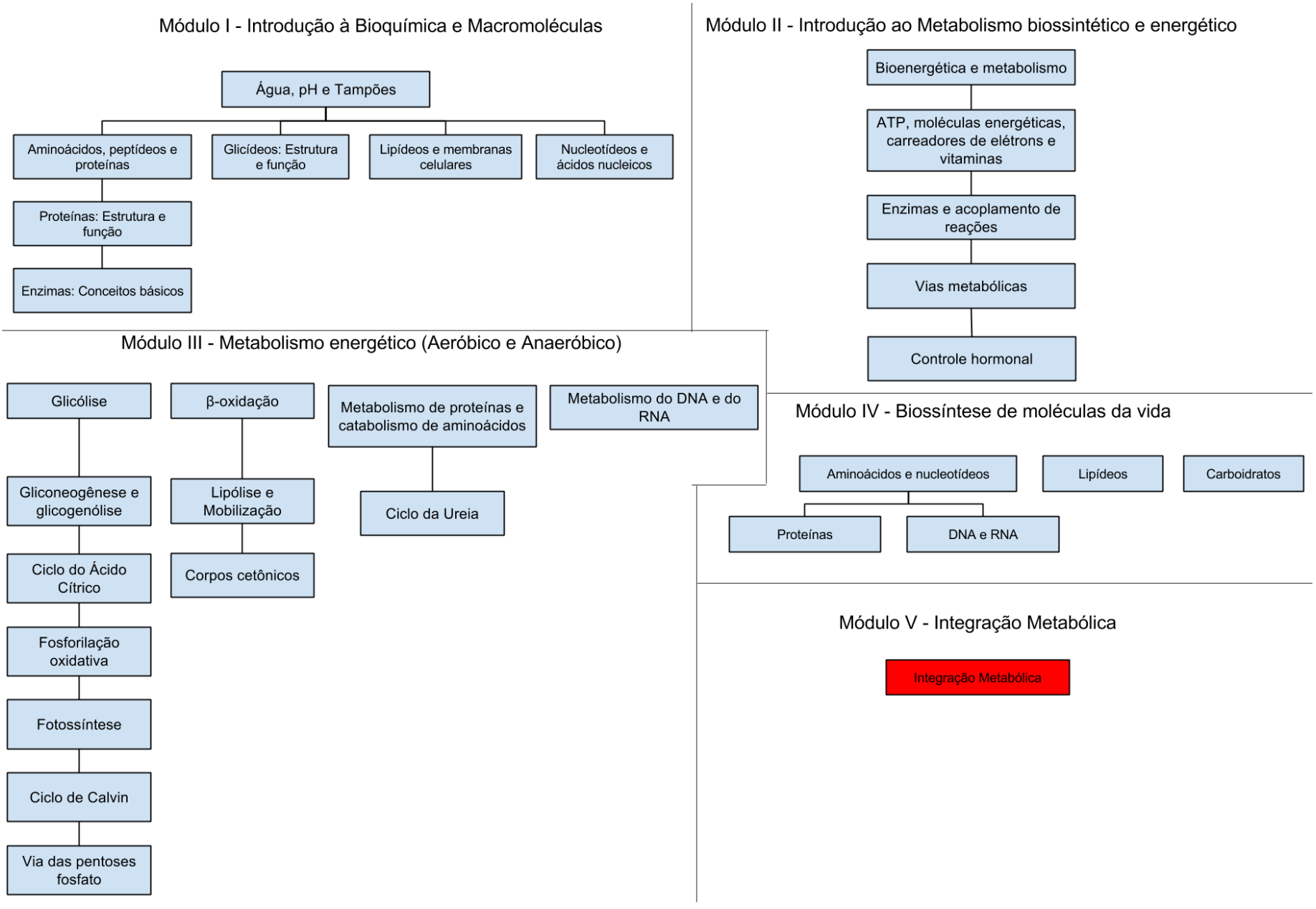


Definição dos Conteúdos, estabelecendo o tempo da disciplina (5 meses, divididos em 32 aulas) e gerando os mapas conceituais. Estes mapas foram divididos em módulos, agregando os temas que foram abordados, conforme pode ser visualizado na Figura 2.

Figura 2: Mapa da definição dos temas por modulo (Fonte: Autores, 2016)

Após a Definição dos Conteúdos, iniciou-se a Fase 2, Definição dos Elementos de Jogos, onde o docente selecionou as mecânicas que mais se adaptavam ao seu plano de ensino, gerando uma série de dinâmicas. Baseando-se na Tabela 1, o docente da disciplina determinou que seu plano de ensino utilizaria as seguintes mecânica: Pressão (temporal), Oportunidades, Atividades Cognitivas, Competição, Níveis, Progresso, Cooperação, Aleatoriedade, Pontos e Economia.

Haviam dinâmicas que foram aplicadas em toda a extensão da disciplina (como elementos de Economia e Progresso) e algumas que eram específicas para algumas atividades, um exemplo pode ser observado na Tabela 4.

Tabela 4: Exemplo da dinâmica Lista de Exercícios Individuais

Nome da ação: Lista de exercícios individual $\quad$ Duração: Final de cada módulo

Descrição: Será aplicada uma lista de exercícios, sendo resolvida individualmente. Ao final, será atribuído um número de pontos baseado na correção da lista.

\section{Propriedades:}

Pressão temporal: Data de entrega sem prorrogação

Oportunidades: Todos os alunos com acesso as mesmas questões

Atividades cognitivas: Exercícios da lista

Competição: Mapa do progresso coletivo, mostrando todos os alunos

Feedback:

Níveis: As listas são interdependentes, a entrega de uma é necessária para receber a próxima

Progresso: Mapa de progresso individual

Pontos: A pontuação da lista será atribuída a nota do aluno

\section{Comentários / Acompanhamento:}

Regra para distribuição de pontos: Cada acerto na lista rende ao aluno 1 ponto, que pode ser utilizado no mercado individual, ou coletivo (Ação que engloba toda a disciplina).

Em seguida, iniciou-se a fase de Implantação. No início desta fase, foi explanado aos alunos as regras geradas e as ações que seriam desenvolvidas juntamente com o cronograma da disciplina. Também foi questionado se todos estavam de acordo, aqueles que não concordaram com a abordagem da disciplina seguiriam um método tradicional, sem aplicação das ações gamificadas. No entanto, todos os alunos que atenderam a disciplina $(\mathrm{N}=24)$ concordaram em participar do processo gamificado. 
V Congresso Brasileiro de Informática na Educação (CBIE 2016)

Anais do XXII Workshop de Informática na Escola (WIE 2016)

Por fim, foi aplicado o Intrinsic Motivation Inventory (IMI) para medir a motivação dos discentes, a fim de avaliar se o processo obteve o efeito engajador que objetivava. Este questionário visa identificar certos aspectos relacionados a motivação do indivíduo, através de uma escala Lickert de 7 itens onde 1 significa "Não é verdade" e 7 significa "Totalmente verdade". Estes aspectos são divididos em grupos, a saber: Interesse/Aproveitamento, Competência Percebida, Importância/Esforço, Pressão/Tensão, Escolha Percebida, Valor/Utilidade e Relacionamento. O questionário utilizado neste estudo foi construído com 21 questões, sendo aplicado e preenchido de forma manual (papel e caneta), ao fim da disciplina, abrangendo perguntas dos grupos: (A) Interesse/Aproveitamento, (B) Competência Percebida, (C) Pressão/Tensão e (D) Escolha Percebida. Para analisar os resultados coletados, foi considerada a média $(\mu)$ das respostas (Tabela 5).

Tabela 5: Perguntas do IMI e a média $(\mu)$ por gênero

\begin{tabular}{|c|c|c|c|c|}
\hline ID & Grupo & Pergunta & $\mu(F)$ & $\mu(M)$ \\
\hline $\mathrm{P} 1$ & A & $\begin{array}{l}\text { Enquanto eu estava fazendo a atividade, eu estava } \\
\text { pensando o quanto estava aproveitando }\end{array}$ & 4,1 & 3,8 \\
\hline $\mathrm{P} 2$ & $\mathrm{C}$ & Eu não me senti nervoso(a) enquanto fazia a atividade. & 4,9 & 4,8 \\
\hline P3 & $\mathrm{C}$ & Eu senti que foi minha escolha fazer essa atividade. & 4,7 & 4,4 \\
\hline $\mathrm{P} 4$ & $\mathrm{~B}$ & Eu acho que sou muito bom nessa atividade. & 3,5 & 3,8 \\
\hline P5 & A & Eu achei essa atividade muito interessante. & 6 & 5,2 \\
\hline P6 & $\mathrm{C}$ & Eu me senti tenso durante essa atividade. & 2,9 & 3,3 \\
\hline $\mathrm{P} 7$ & $\mathrm{~B}$ & $\begin{array}{l}\text { Eu acho que me sai bem nessa atividade, comparado com } \\
\text { outros estudantes. }\end{array}$ & 4,3 & 3,7 \\
\hline P8 & A & Fazer essa atividade foi divertida. & 6 & 5,4 \\
\hline P9 & $\mathrm{C}$ & Eu me senti tranquilo(a) fazendo essa atividade. & 3,8 & 4,2 \\
\hline $\mathrm{P} 10$ & $\mathrm{~A}$ & Eu gostei muito de fazer essa atividade. & 5,2 & 4,2 \\
\hline $\mathrm{P} 11$ & $\mathrm{D}$ & Eu não tive muita escolha em fazer essa atividade. & 3,2 & 4 \\
\hline $\mathrm{P} 12$ & $\mathrm{~B}$ & $\begin{array}{l}\text { Eu estou satisfeito(a) com o meu desempenho nessa } \\
\text { atividade. }\end{array}$ & 4,4 & 4,5 \\
\hline $\mathrm{P} 13$ & $\mathrm{C}$ & Eu estava ansioso(a) durante a atividade. & 3,8 & 3,3 \\
\hline $\mathrm{P} 14$ & A & Eu achei essa atividade muito chata & 1,9 & 2,2 \\
\hline P15 & $\mathrm{D}$ & $\begin{array}{l}\text { Eu senti que eu estava fazendo o que eu queria, enquanto } \\
\text { eu estava fazendo a atividade. }\end{array}$ & 4,6 & 3,6 \\
\hline P16 & $\mathrm{B}$ & Me senti muito competente nesta atividade. & 4,4 & 3,7 \\
\hline $\mathrm{P} 17$ & A & Eu achei a atividade muito interessante. & 6 & 5 \\
\hline $\mathrm{P} 18$ & $\mathrm{C}$ & Eu me senti pressionado enquanto fazia a atividade. & 3,7 & 3,2 \\
\hline P19 & $\mathrm{D}$ & Eu senti obrigação de fazer essa atividade. & 3 & 4,5 \\
\hline $\mathrm{P} 20$ & $\mathrm{~A}$ & Eu descreveria essa atividade como muito agradável. & 4,6 & 5,3 \\
\hline $\mathrm{P} 21$ & $\mathrm{D}$ & Eu fiz essa atividade porque eu não tive escolha. & 3 & 3,2 \\
\hline $\mathrm{P} 22$ & $\mathrm{~B}$ & $\begin{array}{l}\text { Depois de fazer essa atividade por um tempo, me senti } \\
\text { muito competente. }\end{array}$ & 4,9 & 4,1 \\
\hline
\end{tabular}

Dentre os resultados encontrados na Tabela 5, pode-se destacar algumas análises como: Os participantes do gênero feminino refletiram mais sobre seu aproveitamento 
V Congresso Brasileiro de Informática na Educação (CBIE 2016)

Anais do XXII Workshop de Informática na Escola (WIE 2016)

durante o curso $(P 1: \mu(F)=4.1>\mu(M)=3.8$ (dif 0.3)), além de acharem que se saíram melhor que os demais $(P 7: \mu(F)=4.3>\mu(M)=3.7$ (dif0.6)).

Já os participantes do gênero masculino se sentiram menos tranquilos durante a aplicação do método $(P 9: \mu(M)=4.2>\mu(F)=3.8$ (dif 0.4$)$ ), além de sentirem que não tiveram escolha em participar do processo $(P 11: \mu(M)=4.0>\mu(F)=$ 3.2 (dif 0.8)) ou que não estava fazendo o que queria enquanto participava $(P 15: \mu(M)=3.6<\mu(F)=4.6$ (dif 1.0)), além de se sentirem obrigados a terem participado $(P 19: \mu(M)=4.5>\mu(F)=3.0$ (dif 1.5)). Os participantes deste gênero também não se sentiram competentes enquanto realizavam a atividade $(P 16: \mu(M)=3.7<\mu(F)=4.4$ (dif 0.7$)$ ), no entanto se sentiram competentes após o término da mesma $(P 22: \mu(M)=4.1)$. No geral, os alunos acharam que o curso foi muito interessante $(P 5: \mu($ Geral $)=5.6)$ e divertido $(P 8: \mu($ Geral $)=5.7)$.

Por fim, foi realizado uma entrevista com o docente responsável pela disciplina, visando coletar informações para melhorar o processo. De acordo com as respostas coletadas, o docente informou que utilizou uma planilha eletrônica para controlar os elementos que foram implantados, e que a presença de uma ferramenta computacional mais eficiente (ou direcionada) poderia auxiliar neste processo. Também foi exposta a satisfação do docente e que ele planeja utilizá-lo novamente em aulas futuras.

Sobre as discussões do trabalho, em relação ao trabalho realizado por [Hanus and Fox 2014], pode-se observar que o conjunto de elementos utilizados neste estudo influenciou positivamente na motivação e satisfação dos alunos. No entanto, não foi avaliado se a performance deste grupo foi influenciada, ou a comparação social.

Em relação ao estudo realizado por [Mesquita et al. 2013, 2014], o método desenvolvido proporcionou a avaliação da motivação e satisfação dos alunos, encontrando resultados satisfatórios $(\mu($ Grupo $A)=4.6)$, utilizando mecânicas similares, porém com um processo mais detalhado e que aborda o uso de materiais desenvolvidos pelos docentes.

Outra observação pertinente é quanto a aceitação pelo gênero, uma vez que, conforme visualizado no trabalho de [Pedro et al. 2015], os participantes do gênero masculino tiveram uma performance maior que os do feminino. No escopo deste estudo, as mulheres tiveram uma aceitação maior que os rapazes. Isto pode ter sido influenciado pela seleção do conjunto de elementos de gamificação.

Outro fator que pode ter influenciado na diferença é a idade, uma vez que os perfis dos participantes deste trabalho são de alunos do ensino superior, enquanto que no outro trabalho são crianças. Por fim, relacionado os dois estudos, os participantes do primeiro estudo interagiram diretamente com um sistema, desenvolvido pelos autores, sem a intervenção de um professor, enquanto este foi aplicado diretamente em sala de aula, havendo a intervenção do docente responsável pela disciplina.

Conforme pode ser observado nos demais trabalhos, não foi o foco deste avaliar a performance dos alunos, podendo ser explorado em trabalhos futuros. O feedback do docente também foi importante para auxiliar na evolução do método, através da construção de uma ferramenta computacional adaptativa para os tipos de elementos que forem selecionados. 
V Congresso Brasileiro de Informática na Educação (CBIE 2016)

Anais do XXII Workshop de Informática na Escola (WIE 2016)

\section{Conclusões e Trabalhos Futuros}

Este trabalho apresentou uma proposta de método para gamificar aulas e/ou cursos no ensino superior, utilizando planos de aula e ementas como base. O processo foi utilizado em uma disciplina de Bioquímica do curso de Biotecnologia. Os resultados encontrados demonstram que o método foi considerado divertido e interessante, e que grande parte dos alunos se sentiram satisfeitos enquanto participavam do curso.

Uma das principais contribuições do trabalho é a aplicação do método em um curso de Ciências Biológicas, visto que a maioria das aplicações realizadas são nas áreas de Computação e Engenharia, com um perfil de discentes mais propício aos elementos de jogos presentes na gamificação.

Além disso, acredita-se que este trabalho também contribua diretamente para a área de ensino de modo a fornecer uma estratégia para o uso do processo de gamificação em conjunto com planos de aula, ementas e outros documentos organizacionais, produzidos pelo professor. Deste modo, facilitando a implantação do processo nas aulas ministradas.

Por fim, os elementos customizáveis apresentados no modelo permitem que este se adapte a diversos públicos, visto que este é uma das grandes dificuldades de trabalhar com o processo em um ambiente heterogêneo, onde não se conhece o perfil de cada aluno. Deste modo, é possível estudar os efeitos que os elementos implementados implicam nos discentes.

Como trabalhos futuros, espera-se melhorar a formalização do método, assim como aplicar uma metodologia mais específica para o desenvolvimento de atividades colaborativas, visando os objetivos educacionais e suportada pela Teoria da Autodeterminação. Além disso, também espera-se implantar o processo em outras áreas do conhecimento, a fim de explorar os perfis de alunos e elementos que são mais propícios a engajá-los. Por fim, espera-se poder realizar um estudo longitudinal a fim de verificar se o método influência de forma positiva na performance dos alunos.

\section{Referências}

Andrade, F. R., Mizoguchi, R., and Isotani, S. (2016, June). The Bright and Dark Sides of Gamification. In International Conference on Intelligent Tutoring Systems, LNCS 9684, pp. 176-186.

Aparicio, A., Vela, F., Sánchez, J. and Montes, J. (2012). Analysis and application of gamification. In Proceedings of the 13th International Conference on Interacción Persona-Ordenador.

Borges, S. de S., Durelli, V. H. S., Reis, H. M. and Isotani, S. (22 nov 2013). Gamificação Aplicada à Educação: Um Mapeamento Sistemático. Anais do Simpósio Brasileiro de Informática Educativa, n. July 2015, p. 234-243.

Deci, E. L. and Ryan, R. M. (1985). Intrinsic Motivation and Self-Determination in Human Behavior. Boston, MA: Springer US.

Deterding, S., Sicart, M., Nacke, L., O’Hara, K. and Dixon, D. (2011). Gamification. using game-design elements in non-gaming contexts. Proceedings of the 2011 annual conference extended abstracts on Human factors in computing systems - CHI EA '11, p. 2425 . 
V Congresso Brasileiro de Informática na Educação (CBIE 2016)

Anais do XXII Workshop de Informática na Escola (WIE 2016)

De Paula, B. H. ; Valente, J.A. ; Burn, A. . Game-Making as a means to deliver the new computing curriculum in England. Currículo sem Fronteiras , v. 14, p. 46-69, 2014.

Dicheva, D., Dichev, C., Agre, G. and Angelova, G. (2015). Gamification in Education : A Systematic Mapping Study. Educational Technology \& Society, v. 18, n. 3, p. 1-14.

Dignan, A. (2011). Game Frame. Free Press.

Hamari, J., Koivisto, J. and Sarsa, H. (2014). Does Gamification Work? - A Literature Review of Empirical Studies on Gamification. 2014 47th Hawaii International Conference on System Sciences,

Hanus, M. D. and Fox, J. (sep 2014). Assessing the effects of gamification in the classroom: A longitudinal study on intrinsic motivation, social comparison, satisfaction, effort, and academic performance. Computers \& Education, v. 80, p. 152-161.

Huotari, K. and Hamari, J. (2012). Defining gamification: a service marketing perspective. Proceeding of the 16th International Academic MindTrek Conference, p. 1722.

Isotani, S., Bittencourt, I. I., Mizoguchi, R., Costa, E (2009). Estado da Arte em Web Semântica e Web 2.0: Potencialidades e Tendências da Nova Geração de Ambientes de Ensino na Internet. Revista Brasileira de Informática na Educação, v. 17, p. 30-42.

Kapp, K. M. (1 may 2012). The Gamification of Learning and Instruction: Game-based Methods and Strategies for Training and Education.

Mesquita, M. A. A., Toda, A. M., Brancher, J. D. and Do Carmo, R. M. C. (5 mar 2014). Utilizing gamification with social network to aid students in programming languages lessons in higher education IT courses (abstract only). In Proceedings of the 45th ACM technical symposium on Computer science education - SIGCSE '14. . ACM Press.

Mesquita, M., Toda, A., Brancher, J. and Do Carmo, R. (2013). Utilizing Gamification concepts tied with Social Networks to support students in programming classes. Proceedings of the XV Simpósio Internacional de Informática Educativa, p. 127-132.

Mora, A., Riera, D., Gonzalez, C. and Arnedo-Moreno, J. (sep 2015). A Literature Review of Gamification Design Frameworks. In 2015 7th International Conference on Games and Virtual Worlds for Serious Applications (VS-Games). . IEEE.

Pedro, L. Z., Lopes, A. M. Z., Prates, B. G., Vassileva, J., Isotani, S (2015). Does gamification work for boys and girls?. In: Proceedings of the 30th Annual ACM Symposium on Applied Computing - SAC '15. New York: ACM Press. p. 214-219.

Takahashi, R. T. and Fernandes, M. de F. P. (2004). Plano De Aula: Conceito E Metodologia. Acta Paui Enf., v. 17, n. 1, p. 114.

Thiebes, S., Lins, S. and Basten, D. (2014). Gamifying information systems A synthesis of gamification mechanics and dynamics. Twenty Second European Conference on Information Systems, p. 1-17.

Zichermann, G. and Cunningham, C. (2011). Gamification by Design: Implementing Game Mechanics in Web and Mobile Apps. O'Reilly Media; 1 edition. 